\title{
Effects of tsao-ko, Turmeric and Garlic on Body Fat Content and Plasma Lipid Glucose and Liver Lipid Levels in Mice (A comparative Study of Spices)
}

\author{
Longquan $\mathrm{Yu}^{1 *}$ and Hiramitsu SuZUKI ${ }^{2,3}$ \\ ${ }^{1}$ United Graduate School of Agricultural Science, Tokyo University of Agriculture and Technology, 3-5-8, Saiwai, Fuchu-shi, Tokyo, \\ 183-8509, Japan \\ ${ }^{2}$ Kagawa Nutrition University, 3-9-21, Chiyoda, Sakado, Saitama, 350-0288, Japan \\ ${ }^{3}$ National Food Research Institute, 2-1-12, Kannondai, Tsukuba, Ibaraki, 305-8642, Japan
}

Received August 1, 2006; Accepted May 10, 2007

\begin{abstract}
The purpose of this study was to compare the long-term effects of small intakes of tsao-ko, turmeric and garlic on the levels of body fat, plasma lipids, lipid peroxides, glucose and liver lipids in adult mice. Six groups $(n=8$ per group) of adult male mice ( 25 weeks old) were fed a diet containing $1 \%$ or $0.1 \%$ tsao-ko (Amomum tsao-ko Crevost et Lemair), turmeric or garlic powder for 90 days. The final percent change in body weight tended to be lower in mice in the tsao-ko diet group than that in turmeric diet group. There was a significant reduction in plasma and liver triacylylglycerol concentrations in mice on the $0.1 \%$ tsao-ko diet compared with those in the $0.1 \%$ garlic diet group. Plasma glucose concentrations were significantly lower in animals on the $1 \%$ tsao-ko and garlic diets compared with those on the $1 \%$ turmeric diet. The hypolipidemic and hypoglycemic effects of tsao-ko were more pronounced than those of turmeric or garlic.
\end{abstract}

Keywords: Tsao-ko, Turmeric, Garlic, Amomum tsao-ko Crevost et Lemair, Lipids, Glucose, Plasma, Liver, Body fat, Mice

\section{Introduction}

Spices are natural food additives that contribute substantially to the flavor of food and have been used in this context for thousands of years (Atanu et al., 2005; Krishnapura, 2005; Agarwal, 1996). It has also been recognized that spices possess medicinal properties. They have been used effectively in indigenous medicine in India and other countries (Krishnapura, 2005; Agarwal, 1996), and in traditional Chinese medicine, where herbal remedies are classified into categories of low, intermediate and superior; spices are almost exclusively in the superior category. A more detailed understanding of the many advantages of spices in nutrition and medicine is required to enable their full potential to be exploited.

There is now a growing body of scientific evidence to support the concept that spices have medicinal properties and may not only alleviate symptoms, but also help to prevent diseases (Krishnapura, 2005; Reaven et al., 1983). For example, it has been demonstrated that curcumin, the active principle of turmeric, modulates pathological angiogenesis (Gururaj et al., 2002) and a number of spices (garlic, ginger, onion, mint, cloves, cinnamon and pepper) have potential health benefits due to their antioxidant activity in inhibiting lipid peroxidation (Shobana and Naidu, 2000). Despite these advances information on the role of spices in reducing risk factors for various liferelated diseases such as heart disease and diabetes is

* To whom correspondence should be addressed.

E-mail: ylq29@affrc.go.jp limited (Lai and Roy, 2004; Rahman and Lowe, 2006; Westerterp-Plantenga et al., 2006).

Tsao-ko (Amomum tsao-ko Crevost et Lemair), turmeric (Curcuma longa) and garlic (Allium sativum) are among the most commonly used spices in Chinese food, and are also used in traditional Chinese medicine as a natural remedy for many ailments. Unlike many traditional medicines, their lack of toxicity has been clearly demonstrated in both mice and rats (Jang et al., 1992; Chi et al., 1982). Additionally, there is considerable evidence for the positive benefits of turmeric and garlic in reducing risk factors for various life-related diseases (Krishnapura, 2005; Gururaj et al., 2002; Shobana and Naidu, 2000), but until now, there have been no reports on the physiological effects of tsao-ko.

The last 25 years have seen a great increase in the incidence of obesity, both in the Western world and in developing third-world countries (Clapham et al., 2001), and this has been associated with insulin resistance leading to an increase Type- 2 diabetes. This disease is the result of an imbalance between energy intake and usage in the body (Zhao et al., 2005) and it may be that dietary agents that can reduce body fat and plasma glucose levels will help to control this epidemic. It is also conceivable that the flavoring aspect of spices that help redress this imbalance could be used in obesity prevention programs that take into account the emotional and social cues related to food intake (Caroli and Lagravinese, 2002). Our current research effort is aimed at finding new agents that not only achieve body weight control but also im- 
prove metabolic and cardiovascular functions, thereby helping to prevent lifestyle-related diseases. In a previous study, we demonstrated that, in mice, a diet containing $1 \%$ tsao-ko spices resulted in a decrease in body weight, and had hypoglycemic and antioxidant effects (data not shown). The purpose of the present study was to compare the effects of tsao-ko, ingested at the low levels used in Chinese food, with those of turmeric and garlic, on percent change in body weight, body fat content, plasma lipid and glucose concentrations, and the liver lipid content in adult mice.

\section{Materials and Methods}

Diets The spices added to each of the 6 diets were as follows: (1) $1 \%$ tsao-ko, (2) $0.1 \%$ tsao-ko, (3) $1 \%$ turmeric, (4) $0.1 \%$ turmeric, (5) $1 \%$ garlic and (6) $0.1 \%$ garlic. The compositions of the experimental diets were as follows: $47.8 \%$ ( $1 \%$ groups) or $48.7 \%$ (0.1\% groups) corn starch, $20 \%$ casein, $15 \%$ sucrose, $6 \%$ lard, $5 \%$ cellulose powder, $4 \%$ salt mixture, $2 \%$ vitamin mixture and $0.2 \% \mathrm{~L}$ methionine and $1 \%$ or $0.1 \%$ spice. The turmeric and garlic powder was obtained from GABAN Co., Ltd. (Tokyo, Japan). The origin of the turmeric was Malaysia, and garlic was China. The dried fruits of tsao-ko were purchased at the Liouzhou herbal market in Guangxi, China. The skins of the dried tsao-ko were removed, and the fruit was crushed using a disintegrator (Ultra Centrifugal Mill, Retsch KG, ZM1) at room temperature. Lard was supplied by NOF Co. Ltd. (Tokyo, Japan). Salt and vitamin mixtures were purchased from Oriental Yeast Co., Ltd. (Tokyo, Japan). The experimental diets were made once a month, and stored below $5^{\circ} \mathrm{C}$.

Animals Male mice of the Crlj: CD-1 (ICR) strain (4 weeks old) were obtained from Charles River Japan Inc. (Atsugi, Japan). All animals were switched from a laboratory chow, MF (Oriental Yeast Co., Ltd.) to the experimental diets at 25 weeks of age. Adult mice were randomly divided into 6 groups of 8 animals each and each group was fed the experimental diet for 90 days. Animals had to free access to the experimental diet and water until they were killed. Body weights (BW) were measured once a month. The animals were housed in suspended stainless-steel cages with wire mesh bottoms. The animal room was kept at $24 \pm 0.5^{\circ} \mathrm{C}$ and the relative humidity at 65 $\pm 5 \%$. Room lighting consisted of 12 -hour periods of light and dark. All mice were maintained according to the guidelines for experimental animals of the National Food Research Institute, Japan.

Preparation of Plasma Samples and Liver Homogenates At the end of the feeding regimens, all mice were fasted for 20 hours before being anesthetized with diethyl ether. Blood was then collected from the inferior vena cava with a heparinized syringe and placed in ice-cold tubes. The blood was separated by centrifugation at $900 \mathrm{~g}$ for $20 \mathrm{~min}$ at $4^{\circ} \mathrm{C}$. After collecting the plasma, the livers were removed and homogenized with $1 / 15 \mathrm{~mol} / \mathrm{L}$ phosphatebuffered saline $(\mathrm{pH}=7.4)$ using a Teflon-glass homogenizer. After collecting the plasma and liver, the mouse body was frozen in liquid nitrogen, and the carcasses crushed using a disintegrator (Universal Homogenizer; Tokyo Nihon Seiki Seisakusho Co., Tokyo, Japan). Body samples, plasma samples and liver homogenates were stored at $-30^{\circ} \mathrm{C}$ until required for body fat, lipid and glucose analyses.

Body fat, Lipids and Glucose Analyses Body fat content was determined by the methods of Bligh and Dyer (Bligh and Dyer, 1959). Total cholesterol, triacylglycerol, and phospholipid concentrations in the plasma samples and liver homogenates were determined by the methods of Allain et al. (1974), Spayd et al. (1978) and Takayama et al. (1977), respectively. Plasma non-esterified fatty acid (NEFA), glucose, and thiobarbituric acid reactive substances (TBARS) concentrations were measured by the methods of Shimizu et al. (1979), Trinder (1969) and Yagi (1976), respectively.

Statistical Analysis All results were expressed as mean \pm SE. The statistical significance of differences in lipid components and glucose between the experimental spice groups were determined by one-way analysis of variance (ANOVA) and Spjotvoll/Soline test using the STATISTICA statistical program package (Statsoft, Tulsa, $\mathrm{OK})$; the significance was set at $\mathrm{P}<0.05$.

\section{Results}

Food Intake, Change in Body Weight, and Body Fat There were no marked differences in the average food intake ( $\mathrm{g} /$ mouse/day) between any of the experimental diet groups as follows: $1 \%$ tsao-ko group, $4.59 \pm 0.13 ; 0.1 \%$ tsao-ko group, $4.57 \pm 0.14 ; 1 \%$ turmeric group, $4.56 \pm 0.13$; $0.1 \%$ turmeric group, $4.53 \pm 0.13 ; 1 \%$ garlic group, $4.54 \pm$ 0.13 and $0.1 \%$ garlic group, $4.47 \pm 0.14$. The percent change in body weight of mice after 90 days of dietary intake in the $1 \%$ and $0.1 \%$ of tsao-ko diet groups were significantly lower than those of the $1 \%$ and $0.1 \%$ turmeric diet groups (Figs. 1A, 1B). The slightly lower value of final mean body fat content seen in the $0.1 \%$ tsao-ko diet group compared with that of the $0.1 \%$ of garlic and turmeric groups was not statistically significant (Fig. 1C).

Plasma Lipids, TBARS and Glucose Plasma total cholesterol concentrations were not statistically significantly different between any of the experimental diet groups (Fig. 2A). The significantly lower value for the plasma triacylglycerol concentration observed in the $0.1 \%$ of tsao-ko diet group compared with the $0.1 \%$ garlic diet group (Fig. 2B) was not evident in the corresponding $1 \%$ diet groups. There were no statistically significant differences in plasma phospholipid or NEFA concentrations between any of the $1 \%$ or $0.1 \%$ spice content experimental diet groups (Figs. 2C, 2D). The plasma TBARS concentrations of the $1 \%$ tsao-ko diet group were significantly lower than the $1 \%$ turmeric diet group, but the differences between the corresponding $0.1 \%$ spice supplemented diet groups did not achieve statistical significance (Fig. 2E). The $1 \%$ of tsao-ko supplemented diets also resulted in a significant reduction in the plasma glucose concentrations compared with $1 \%$ turmeric diet (Fig. 2F), but again, this was not evident in the $0.1 \%$ spice supplemented diet groups.

Liver lipids Total cholesterol content in the liver 


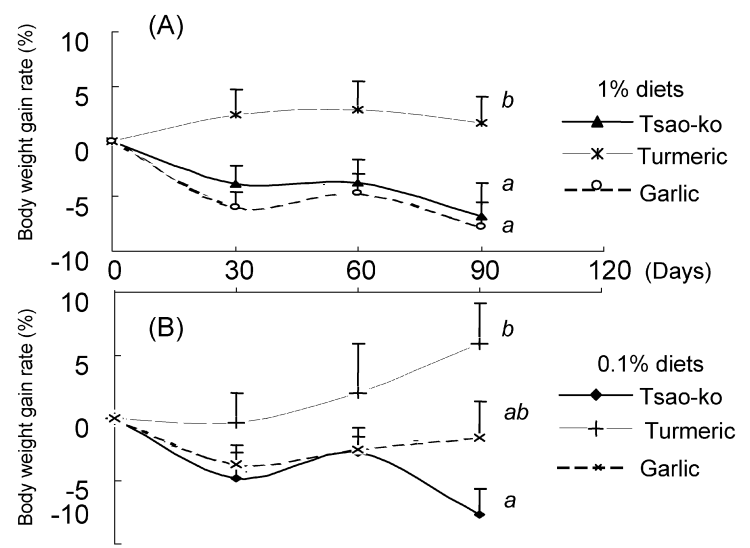

(C)

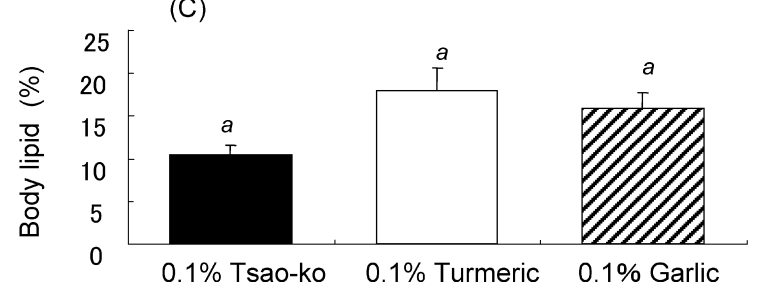

Fig. 1. Body weight gain rate and body lipid content in mice fed diets supplemented with spice $(n=8)$. Values are means $\pm \mathrm{SE}$. The statistical significance of differences between each dietary groups were determined at $P<0.05$ by the Spjotvoll/Stoline test. was not statistically significantly different between any of the experimental diet groups (Fig. 3A). The statistically significant reduction observed in liver triacylglycerol levels in the $0.1 \%$ tsao-ko diet group compared with the $0.1 \%$ garlic diet group (Fig. $3 \mathrm{~B}$ ), was not apparent in the corresponding $1 \%$ spice supplemented diet groups. The phospholipid content of liver from mice fed the $1 \%$ tsaoko diet group was significantly lower than those from animals that were fed the $1 \%$ of garlic and turmeric diet groups, and this difference was not evident in those fed the $0.1 \%$ diets (Fig. 3C).

\section{Discussion}

Tsao-ko, turmeric and garlic are among the most commonly used spices in Chinese food, and are also used in traditional Chinese medicine as a natural remedy for many ailments. We have already discovered that tsaoko has the triacylglycerol-lowering effect, hypoglycemic action, and antioxidant action in screening experiment (Longquan et al., 2006). In addition, garlic and turmeric have been documented as having pronounced hypolipidemic, hypoglycemic and antioxidant influence in a variety of experimental animal systems, garlic and turmeric spices have also been found to be efficacious in human studies (Adachi, et al., 2006; Krishnapura, 2005; Gururaj et al., 2002; Shobana and Naidu, 2000). The present study investigated the physiological regulation ability of tsaoko spice, compared with garlic and turmeric, on the levels of body fat, plasma lipids, lipid peroxides, and glucose, and liver lipids in adult mice.
(A)

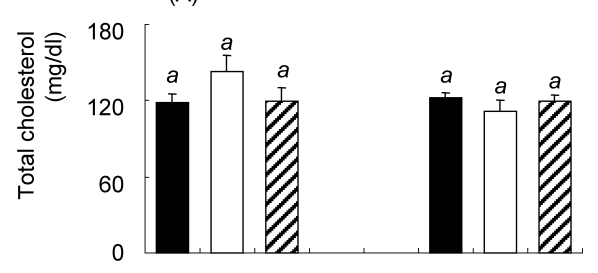

(B)

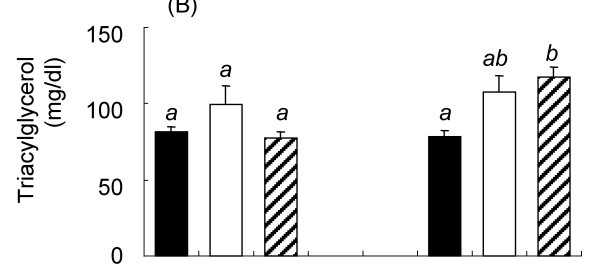

(C)

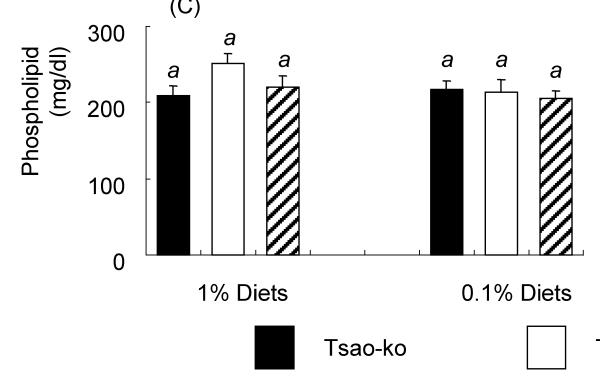

(D)

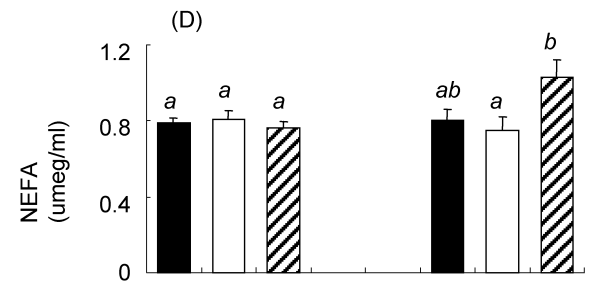

(E)

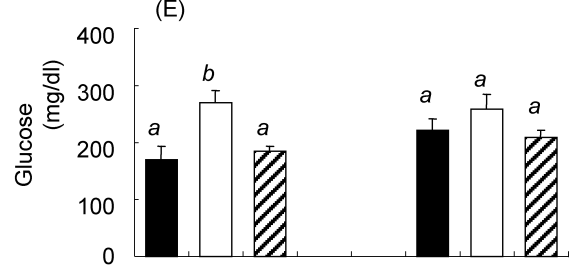

( $\mathrm{F})$

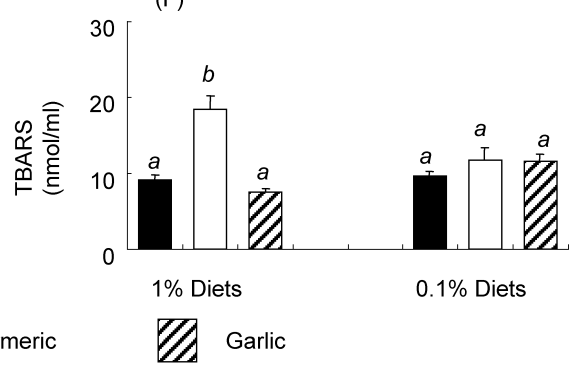

Fig. 2. Plasma total cholesterol, triacylglycerol, phospholipids, NEFA, glucose and TBARS concentrations of mice fed experimental diets for 90 days $(n=8)$. Values are means \pm SE. Values for each sample with different superscript letters in the same lipid class and glucose were significantly different at $P<0.05$ by the Spjotvoll/Stoline test. 

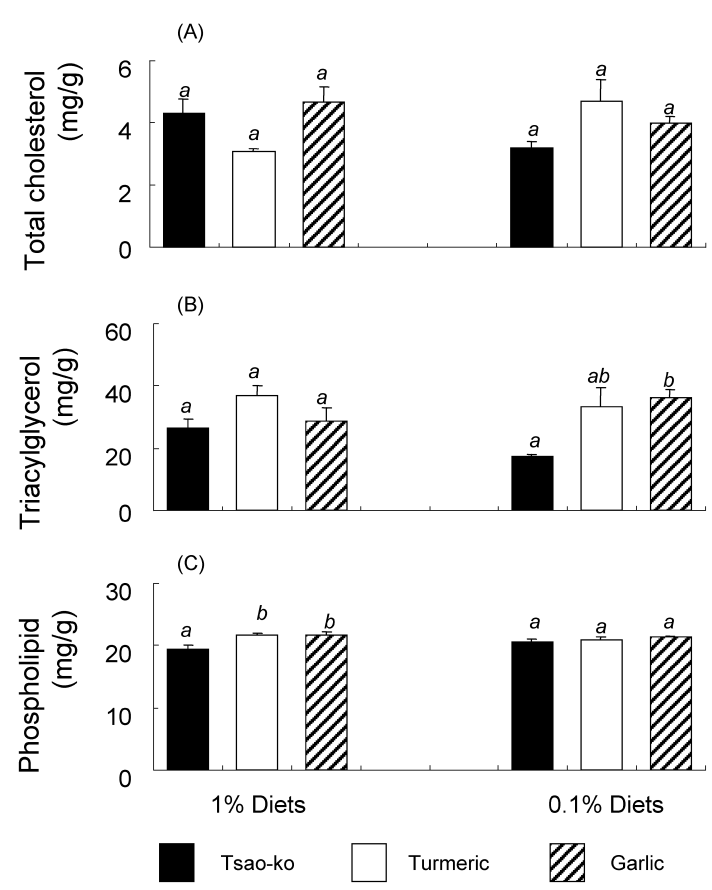

Fig. 3. Total cholesterol, triacylglycerol and phospholipid concentrations of liver in mice fed experimental diets for 90 days $(n=8)$. Values are means \pm SE. Values for each sample with different superscript letters in the same lipid class were significantly different at $P<0.05$ by the Spjotvoll/ Stoline test.

We previously demonstrated that a $1 \%$ tsao-ko diet inhibited increases in body weight compared with a control diet in mice with free access to food and water, and with the same level of locomotion. The present study extends these observations to a comparison of tsao-ko with turmeric and garlic, as the latter two spices are known to inhibit body weight gain. In the adult mice, a $1 \%$ or $0.1 \%$ tsao-ko diet significantly reduced body weight gain compared with mice fed $1 \%$ or $0.1 \%$ turmeric diets (Figs. 1A, 1B). In previous studies high doses of turmeric oleoresin were shown to reduce the weight gain, improve food-conversion efficiency, increase the weight of the liver and thyroid and induce histological changes in the liver, kidney and urinary bladder in pigs (Bille et al., 1985). Oral administration of a higher dose of turmeric (5\%) for a longer duration (90 days) caused a significant reduction in body weight gain in both mice and rats (Deshpande, et al., 1998). In another study, oral administration of an ethanolic extract of turmeric $(100 \mathrm{mg} / \mathrm{kg}$ per day) also inhibited body weight gain and induced significant changes in organ weights in mice (Qureshi et al., 1992). Our results indicate that tsao-ko is even more effective in reducing weight gain than turmeric. This improved efficacy was reflected in the suppressed plasma glucose concentrations observed in mice fed $1 \%$ tsao-ko compared with those fed $1 \%$ turmeric (Fig. $2 \mathrm{~F}$ ). There was also a trend towards a reduction in body lipids in mice fed the $0.1 \%$ tsao-ko diet compared with both turmeric and garlic diets but this was not statistically significant (Fig. 1C).

The $\mathrm{Zn}$ (II) complex ( $\mathrm{Zn}(\mathrm{alx}) 2$ ) of allixin, isolated from dry garlic, significantly improved hyperleptinemia and obesity, and depressed plasma adiponectin levels in diabetic mice (Adachi, et al., 2006). In the present study using adult mice, the effects on weight gain of tsao-ko were similar to those obtained with garlic. This suggests that the effect of tsao-ko in improving obesity in diabetic mice would be similar to that of garlic but this remains to be explored. In our studies, the plasma and liver total cholesterol concentrations were similar in each of the experimental diet groups (Figs. 2A;3A). Plasma and liver triacylglycerol content was lower in mice fed the $0.1 \%$ tsao-ko diet than in those fed either the $0.1 \%$ garlic diet or turmeric diet but this difference was only statistically significant for the tsao-ko and garlic group (Figs. 2 $\mathrm{B} ; 3 \mathrm{~B})$. It is curious that this effect was not apparent in mice fed higher concentrations (1\%) of tsao-ko and garlic. The mechanisms of action of these spices require further elucidation before the reasons for this anomaly can be understood. In other studies turmeric and red pepper and their active principles, curcumin and capsaicin, were found to be effective as hypocholesterolemic agents under conditions of experimentally induced hypercholesterolemia/hyperlipemia in rats (Kempaiah and Srinivasan, 2002; Patil and Srinivasan, 1971; Sambaiah et al., 1982; Subba et al., 1970). A similar action of dietary curcumin was also seen in rats with experimentally induced diabetes (Babu and Srinivasan, 1997). Liver triacylglycerol concentrations of mice fed turmeric extract were reduced to half that observed in controls (Asai, et al., 1999). Garlic has also been shown to reduce cholesterol and triglyceride levels in vivo (Kamanna and Chandrasekhara, 1982; Kempaiah and Srinivasan, 2002; Kempaiah and Srinivasan, 2006), and the beneficial effect was attributed to the essential oil component (Kamanna and Chandrasekhara, 1984). Our results suggest that tsao-ko is at least as effective as turmeric, and possibly more effective than garlic in ameliorating a hyperlipemidic state.

There is currently a strong interest in natural antioxidants, especially those from edible plants, as evidenced by the escalating amount of published research on these agents. The antioxidant properties of curcumin, capsaicin, piperine (from pepper) and eugenol (from cloves) have been established by measuring lipid peroxidation in vivo (rat tissues) and in vitro (Joe and Lokesh, 1994; Reddy and Lokesh, 1992, Kempaiah and Srinivasan 2004). Garlic has also been shown to inhibit lipid peroxidation and exhibit scavenging activity in isolated rat hepatocytes (Nuutila et al., 2003). Turmeric, in particular, has been shown to exhibit pharmacological effects including antitumour, anti-inflammatory, antioxidant and anti-infectious activities (Ramírez-Tortosa et al., 1999), and to reduce ironinduced hepatic damage by lowering lipid peroxidation (Pulla-Reddy and Lokesh, 1996). In the present study, the significantly lower plasma TBARS concentrations in the $1 \%$ tsao-ko diet group compared with the $1 \%$ turmeric diet group (Fig. 2E) suggest that, at the higher concentration of supplements, the antioxidant action of tsao-ko was more effective than that of turmeric. In another study, tsao-ko extracts were also shown to have higher antioxi- 
dant activity than a-tocopherol or butylated hydroxytoluene (Martin, et al., 2000).

Decreases in plasma glucose concentrations observed following an intake of garlic or turmeric (Thomson, et al., 2006; Suresh-Kumar et al., 2005) may be a consequence increased insulin secretion and a greater sensitivity to insulin (Shirai and Suzuki, 2004). For example, in diabetic rats, an $18 \%$ improvement in fasting blood glucose levels were observed following dietary supplementation with turmeric (Suresh-Kumar et al., 2005). Garlic has long been believed to possess a hypoglycemic effect (Agarwal, 1996; Augusti, 1996), via a similar mechanism of action (Cheng-Tzu et al., 2005; El-Demerdash et al., 2005). It has also been demonstrated that the antidiabetic effect of garlic extract is more effective than that observed with the antidiabetic drug glibenclamide in diabetic rats (Eidi et al., 2006). It is evident from our results that the hypoglycemic action following dietary supplementation with $1 \%$ tsao-ko and $1 \%$ garlic were similar and both were likely to be more effective than turmeric in ameliorating a hyperglycemic state (Fig. $2 \mathrm{~F}$ ).

In conclusion, in this 12 -week comparative study in mice of dietary supplementation with three spices, tsao-ko was at least as effective, and in some aspects more effective, than either garlic or turmeric in maintaining a metabolically balanced state. In particular, the triacylglycerollowering effects of tsao-ko were more pronounced than those of garlic even at the $0.1 \%$ level of supplementation. At the low concentration of supplementation, its effects on decreasing body weight gain and reducing blood glucose levels, as well as its antioxidant potential, were greater than those of corresponding levels of turmeric. These results suggest that tsao-ko supplemented diets not only impart flavor to food but possess potential health benefits. They may also play an important role in the prevention of obesity, thereby reducing the risk of lifestyle-related diseases. Further detailed studies in humans and in noninsulin dependent diabetes mellitus animal models are necessary to confirm these proposed benefits.

\section{References}

Adachi, Y., Yoshida, J., Kodera, Y., Kiss, T., Jakusch, T., Enyedy, E.A., Yoshikawa, Y. and Sakurai, H. (2006) Oral administration of a zinc complex improves type 2 diabetes and metabolic syndromes. Biochem. Biophys. Res. Commun., 351, 165-170.

Agarwal, K.C. (1996) Therapeutic actions of garlic constituents. Med. Res. Rev., 16, 111-124.

Allain, C.C., Poon, L.S., Chan, C.S.G., Richmond, W. and Fu, P.C. (1974). Enzymatic determination of total serum cholesterol. Clin. Chem., 20, 470-475.

Asai, A., Nakagawa, K. and Miyazawa, T. (1999) Antioxidative effects of turmeric, rosemary and capsicum extracts on membrane phospholipid peroxidation and liver lipid metabolism in mice. Biosci. Biotechnol. Biochem. 63, 2118-2122.

Atanu, B., Beena, M., Liang, S., Hari, M., Kadam, R.M., Dutta, S., Hong-Yu, Z. and Priyadarsini, K.I. (2005) Evaluation of a new copper (II)-curcumin complex as a superoxide dismutase mimic and its free radical reactions. Free Radical. Bio. Med., 39, 811822.

Augusti, K.T. and Sheela, C.G. (1996).Antiperoxide effect of S-allyl cysteine sulfoxide, an insulin secretagogue, in diabetic rats.
Experientia. 52, 115-120.

Babu, P.S. and Srinivasan, K. (1997) Hypolipidemic action of curcumin, the active principle of turmeric (Curcuma longa) in streptozotocin induced diabetic rats. Mol. Cell. Biochem., 166, 169-175.

Bille, J.C., Hansen, E.V. and Wurtzen, G. (1985) Subchronic oral toxicity of turmeric oleoresin in pigs. Food Chem. Toxicol., 23, 967-973

Bligh, E. G. and Dyer, W.J. (1959) A rapid method of total lipid extracton and purification. Can. J. Biochem. Physiol., 37, 911917.

Caroli, M. and Lagravinese, D. (2002) Prevention of obesity. Nutr. Res., 22, 221-226.

Cheng-Tzu, L., Hunry, H., Chong-Kuei, L., Phi-Sam, C. and LeeYan, S. (2005) Effects of garlic oil and diallyl trisulfide on glycemic control in diabetic rats. Eur. J. Pharmacol., 516, 165173.

Chi, M.S., Koh E.T. and Stewart, T.J. (1982) Effects of garlic on lipid metabolism in rats fed cholesterol or lard. J. Nutr., 112, 241248.

Clapham, J.C., Arch, J.R. and Tadayyon, M. (2001) Anti-obesity drugs: a critical review of current therapies and future opportunities. Pharmacol. Ther. 89, 81-121.

Deshpande, S.S., Lalitha, V.S., Ingle, A.D., Raste, A.S., Gadre, S.G. and Maru, G.B. (1998) Subchronic oral toxicity of turmeric and ethanolic turmeric extract in female mice and rats. Toxicology. Letters., 95, 183-193.

Eidi, A., Eidi, M., and Esmaeili, E. (2006) Antidiabetic effect of garlic (Allium sativum $\mathrm{L}$ ) in normal and streptozotocin-induced diabetic rats. Phytomedicine., 13, 624-662.

El-Demerdash, F.M., Yousef, M.I. and Abou, N.I. (2005) Biochemical study on the hypoglycemic effects of onion and garlic in alloxan-induced diabetic rats. Food. Chem. Toxicol., 43, 57-63.

Gururaj, A.E., Belakavadi, M., Venkatesh, D.A., Marme, D. and Salimath, B.P., (2002) Molecular mechanisms of the antiangiogenic effect of curcumin, Biochem. Biophys. Res. Commun., 297, 934-942.

Jang, J.J., Dever, D.E., Logsdon, D.L. and Ward, J.M. (1992) A 4week feeding study of ground red chilli (Capsicum annuum) in male B6C3F1 mice, Food Chem. Toxicol., 30, 783-787.

Joe, B. and Lokesh, B.R. (1994) Role of capsaicin, curcumin and dietary $\mathrm{n}-3$ fatty acids in lowering the generation of reactive oxygen species in rat peritoneal macrophages, Biochim. Biophys. Acta., 1224, 255-263.

Kamanna, V.S. and Chandrasekhara, N. (1982) Effect of garlic (Allium sativum Linn.) on serum lipoprotein and lipoprotein cholesterol levels in albino rats rendered hypercholesterolemic by feeding cholesterol, Lipids., 17, 483-488.

Kamanna, V.S. and Chandrasekhara, N. (1984) Hy pocholesteremic activity of different fractions of garlic. Indian. J. Med. Res., 79, 580-583.

Kempaiah, R.K. and Srinivasan, K. (2002) Integrity of erythrocytes of hypercholesterolemic rats during spices treatment. Mol. Cell. Biochem., 236, 155-161.

Kempaiah, R.K. and Srinivasan, K. (2004) Influence of dietary curcumin, capsaicin and garlic on the antioxidant status of red blood cells and the liver in high-fat-fed rats, Ann. Nutr. Metab., 48, 314-320.

Kempaiah, R.K. and Srinivasan, K. (2006). Beneficial influence of dietary curcumin, capsaicin and garlic on erythrocyte integrity in high fat fed rats, J. Nutr. Biochem., 17, 471-478.

Krishnapura, S. (2005) Spices as influencers of body metabolism: an overview of three decades of research, Food Res. Int., 38, 7786.

Lai, P.K. and Roy, J. (2004). Antimicrobial and chemopreventive properties of herbs and spices, Curr. Med. Chem., 11, 1451-1460.

Longquan, Y.; Nobuya, S. and Hiramitsu, S. (2006) Effects of some 
chinese spices on body weights, plasma lipids, lipid peroxides, and glucose, and liver lipids in mice. Food Sci. Technol. Res., 13, 155-161.

Martin, T. S.; Kikuzaki, K.; Hisamoto, M.; Nakatani, N. Constituents of Amonum tsao-ko and their radical scavenging and antioxidant activities. J. Am. Oil. Chem. Soc., 2000, 77, 667-73.

Nuutila, A.M., Puupponen-Pimiä, R., Marjukka, A. and OksmanCaldentey, K.M. (2003) Comparison of antioxidant activities of onion and garlic extracts by inhibition of lipid peroxidation and radical scavenging activity. Food Chem., 81, 485-493.

Patil, T.N. and Srinivasan, M. (1971) Hypocholesterolemic effect of curcumin in induced hypercholesterolemic rats. Indian J. Exp. Biol., 9, 167-169.

Qureshi, S., Shah, A.H. and Ageel, A.M. (1992) Toxicity studies on Alpinia galanga and Curcuma longa. Planta Med., 58, 124-127.

Rahman, K. and Lowe, G.M. (2006). Garlic and cardiovascular disease: a critical review. J. Nutr., 136, 736S-740S.

Ramírez-Tortosa, M.C., Mesa, M.D., Aguilera, M.C., Quiles, J.L., Baró, L., Ramirez-Tortosa, C.L., Martinez-Victoria, E. and Gil, A. (1999) Oral administration of a turmeric extract inhibits LDL oxidation and has hypocholesterolemic effects in rabbits with experimental atherosclerosis. Atherosclerosis., 147, 371-378.

Reaven, E., Wright, D., Mondon, C.E., Solomon, R., Ho, H. and Reaven, G.M. (1983) Effect of age and diet on insulin secretion and insulin action in the rat. Diabetes., 32, 175-180.

Reddy, A.C.P. and Lokesh, B.R. (1992) Studies on spice principles as antioxidants in the inhibition of lipid peroxidation of rat liver microsomes. Mol. Cell. Biochem., 111, 117-124.

Reddy, A.C.P. and Lokesh, B.R. (1996) Effect of curcumin and eugenol on iron-induced hepatic toxicity in rats. Toxicology., 107, 39-45.

Sambaiah, K., Ratankumar, S., Kamanna, V.S., Satyanarayana M. N. and Rao, M.V.L. (1982) Influence of turmeric and curcumin on growth, blood constituents and serum enzymes in rats. $J$. Food Sci. Technol., 19, 187-190.

Shimizu, S., Yasui, K., Tani, Y., and Yamada, H. (1979). Acyl-CoA oxidase from Candida tropicalis. Biochem. Biophys. Res. Commun.,
91, 108-113.

Nobuya, S. and Hiramitsu, S. (2004) Effects of Western, vegetarian and Japanese dietary fat model diets with or without green tea extract on the plasma lipids and glucose, and liver lipids in mice. Ann. Nutr. Metab., 48, 95-102.

Shobana, S. and Naidu, K.A. (2000) Antioxidant activity of selected Indian spices. Prostag. Leukotr. Ess., 62, 107-110.

Spayd, R.W., Bruschi, B., Burdick, B.A., Dappen, G.M., Eikenberry, J.N., Esders, T.W., Fiqueras, J., Goodhue, C.T., Larossa, D.D., Nelson, R.W., Rand, R.N. and Wu, T.W. (1978). Multilayer film elements for clinical analysis: applications to representative chemical determinations. Clin. Chem., 24, 1343-1350.

Subba, D.R., Chandrasekhara, N., Satyanarayana M.N. and Srinivasan, M. (1970) Effect of curcumin in serum and liver cholesterol levels in the rat. J. Nutr., 100, 1307-1315.

Suresh-Kumar, G., Shetty, A.K., Sambaiah, K. and Salimath, P.V. (2005) Antidiabetic property of fenugreek seed mucilage and spent turmeric in streptozotocin-induced diabetic rats. Nutr. Res., 25, 1021-1028.

Takayama, M., Itoh, S., Nagasaki, T. and Tanimizu, I. (1977). A new enzymatic method for determination of serum cholinecontaining phospholipids. Clin. Chim. Acta., 79, 93-98.

Thomson, M., Al-Qattan, K. K., Bordia, T. and Ali, M. (2006) Including garlic in the diet may help lower blood glucose, cholesterol, and triglycerides. J. Nutr., 136, 800S-802S.

Trinder, P. (1969). Determination of blood glucose using an oxidase-peroxidase system with a non-carcinogenic chromogen. J. Clin. Path., 22, 158-161.

Westerterp-Plantenga, M., Diepvens, K., Joosen, A.M., BerubeParent, S. and Tremblay, A. (2006). Metabolic effects of spices, teas, and caffeine. Physiol. Behav. in press.

Yagi, K. (1976) Asimple fluorometric assay for lipoperoxide in blood plasma. Biochem. Med., 15, 212-216.

Zhao, B., Wall, R.J. and Jinzeng, Y. (2005) Transgenic expression of myostatin propeptide prevents diet-induced obesity and insulin resistance. Biochem. Biophys. Res. Commun., 337, 248-255. 